\title{
Sarcoma de Kaposi primário do pênis
}

\author{
Primary Kaposi's sarcoma of the penis
}

\author{
Maira Mitsue Mukai ${ }^{1}$ \\ Lucio Caldas $^{3}$ \\ Jesus Rodriguez Santamaría ${ }^{5}$
}

\author{
Talitha Chaves ${ }^{2}$ \\ José Fillus Neto ${ }^{4}$
}

Resumo: Sarcoma de Kaposi é um tumor vascular que afeta a parede dos vasos linfáticos. Possui quatro formas: clássica, endêmica, iatrogênica e associada ao HIV. É uma doença sistêmica, maligna, multifatorial e de curso variável. A apresentação inicial no pênis é rara, e mais observada em pacientes HIV positivos. Em pacientes HIV negativos, os casos que ocorrem nesta região, apresentam-se com pápulas, nódulos, placas e lesões verruciformes, assintomáticas. Para o tratamento da forma clássica, dispõem-se de excisão cirúrgica, crioterapia, eletrocirurgia, laser e radioterapia. Neste trabalho, é relatado um caso raro de um paciente com a forma clássica, em região peniana tratado com sucesso com radioterapia. Palavras-chave: Pênis, Radioterapia, Sarcoma de Kaposi

\begin{abstract}
Kaposi's sarcoma is a vascular tumor involving the wall of lymphatic vessels. There are four types: classic, endemic, iatrogenic and HIV-associated. It is a systemic, malignant and multifactorial disease and has a variable course. The primary presentation on the penis is uncommon and is mainly observed in HIV-positive patients. In HIV-negative individuals, asymptomatic papules, nodules, plaques and verrucous lesions are found. The treatment for the classic form involves surgery, cryotherapy, electrosurgery, laser and radiation therapy. The authors present a rare case of a patient with the classic form on the penis, who was successfully treated by radiation therapy.

Keywords: Penis; Radiotherapy; Sarcoma, Kaposi
\end{abstract}

\section{INTRODUÇÃO}

Sarcoma de Kaposi é uma neoplasia vascular de origem multifocal, descrita em 1872, por Morris Kaposi. Desde então, foram retratados quatro formas clínicas: clássica, endêmica, iatrogênica e associada ao HIV - sendo essa última a forma mais comum. Já a forma clássica, confinada à região peniana, é extramente rara. Os autores relatam um caso de sarcoma de Kaposi em glande, tratado com sucesso pela radioterapia.

\section{RELATO DO CASO}

Paciente masculino, 64 anos, com história de lesão no meato peniano, com 8 meses de evolução, de cor violácea, associada à alteração do jato urinário, sem outros sintomas associados. Ao consultar o urologista, foi submetido à exérese da lesão, com diagnóstico de hemangioma de meato uretral. Após o procedimento, houve aparecimento de novas lesões, semelhantes à primeira, no pênis, no qual surgia sangramento local (Figura 1). Na palpação, observou-se adenopatia inguinal direita. Foi realizada nova biópsia de uma das lesões, com achados histológicos compatíveis com sarcoma de Kaposi (Figuras 2 e 3).

$\mathrm{Na}$ investigação complementar, foram solicitados VDRL e anti-HIV, ambos negativos. Houve encaminhamento do paciente para tratamento com radioterapia, sendo completado o total de 40 sessões. Ao final, houve diminuição dos nódulos, permanecendo

\footnotetext{
Recebido em 04.04.2008

Aprovado pelo Conselho Consultivo e aceito para publicação em 20.10.08.

* Trabalho realizado no Serviço de Dermatologia do Hospital de Clínicas de Curitiba - Universidade Federal do Paraná (UFPR) - Curitiba (PR), Brasil. Conflito de interesse: Nenhum / Conflict of interest: None

Suporte financeiro: Nenhum / Financial funding: None

Professora substituta de Dermatologia da Universidade Federal do Paraná (UFPR) - Curitiba (PR), Brasil

Aluna de graduação do curso de Medicina da Universidade Federal do Paraná (UFPR) - Curitiba (PR), Brasil.

Médico Dermatologista, membro da Sociedade Brasileira de Dermatologia - Curitiba (PR), Brasil.

Professor Adjunto da Anatomia Patológica da Universidade Federal do Paraná (UFPR) - Curitiba (PR), Brasil.

Professor Adjunto e Chefe do Serviço de Dermatologia da Universidade Federal do Paraná (UFPR) - Curitiba (PR), Brasil.

(C)2009 by Anais Brasileiros de Dermatologia
} 


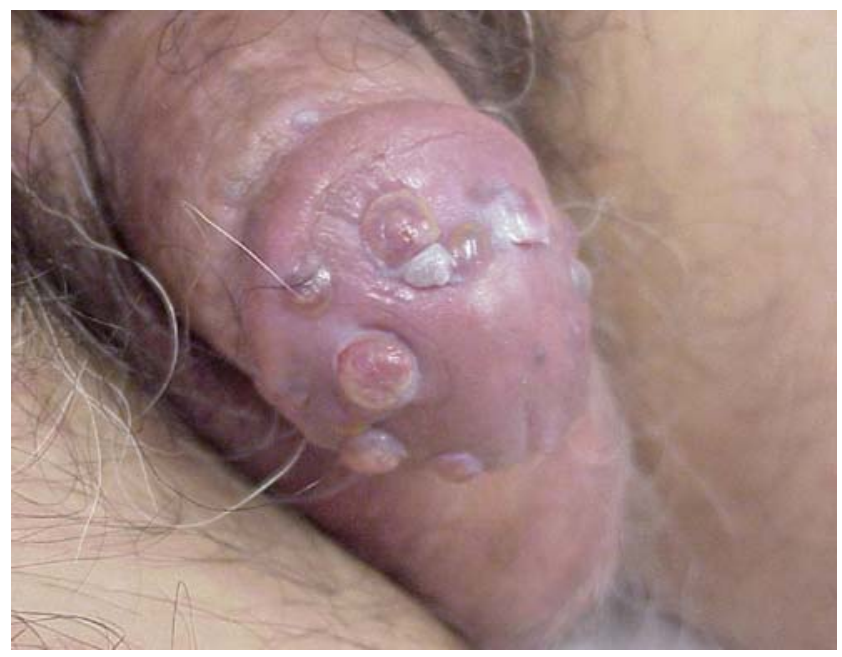

FiguRA 1: Numerosas pápulas e nódulos violáceos agrupados glande peniana

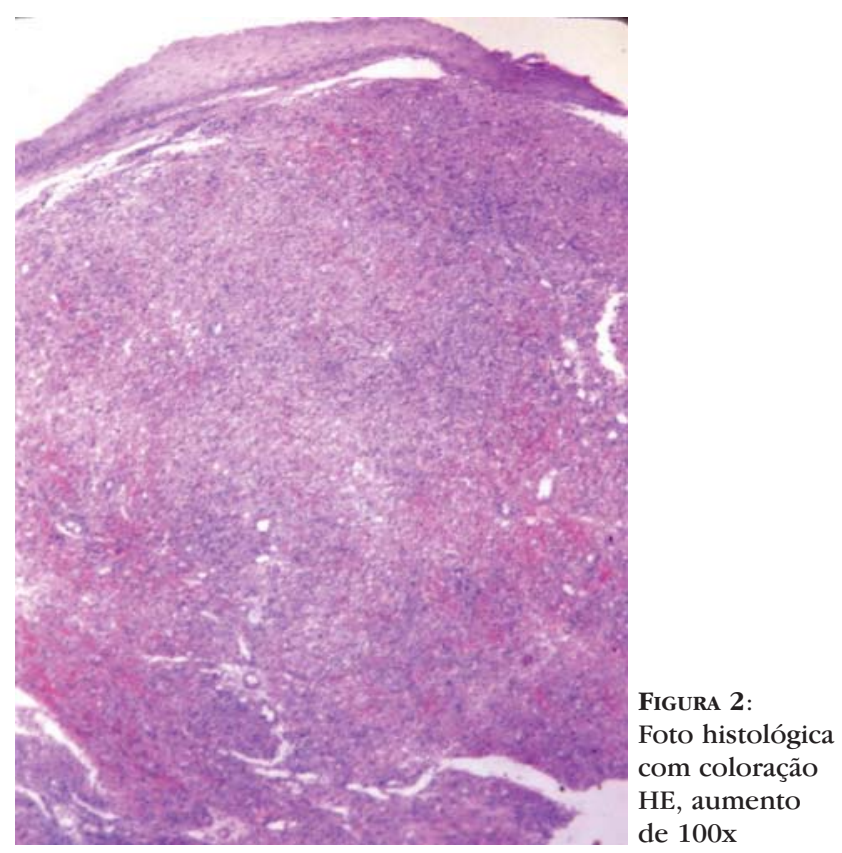

com pequenas ulcerações em sulco bálano-prepucial, em decorrência do tratamento, ocorrendo a cicatrização, após 30 dias (Figuras 4).

\section{DISCUSSÃO}

O sarcoma de Kaposi pode ser classificado em quatro formas distintas: endêmico, iatrogênico, associado à AIDS e clássico. ${ }^{1}$ A forma clássica acomete, frequentemente, pacientes idosos do sexo masculino, de origem do Mediterrâneo e judeus do leste europeu, apresentando-se como doença indolente, com lesões nodulares na pele, atingindo mais comumente a parte inferior das pernas e pés, e raramente apresentam envolvimento visceral.

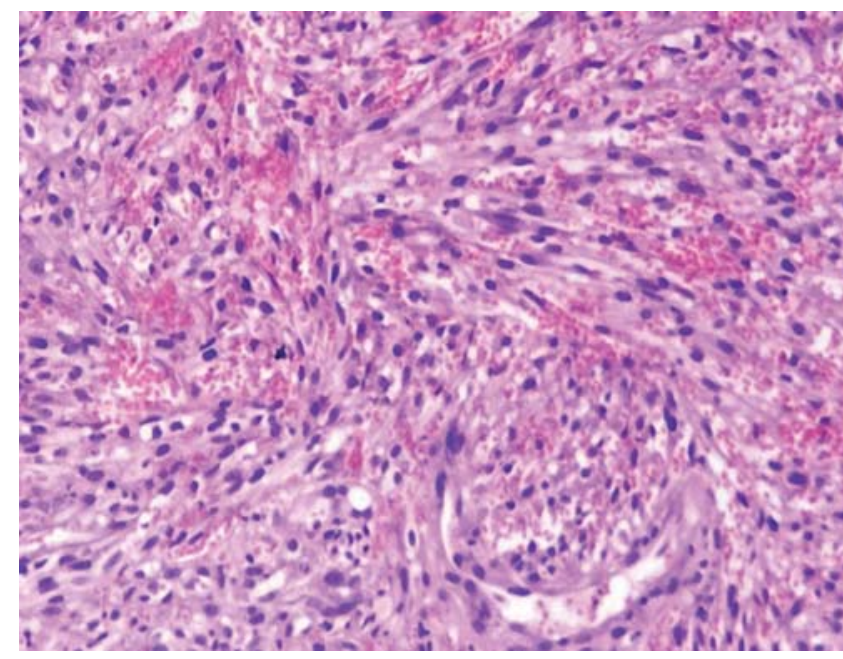

Figura 3: Foto histológica com coloração HE, aumento de 400x
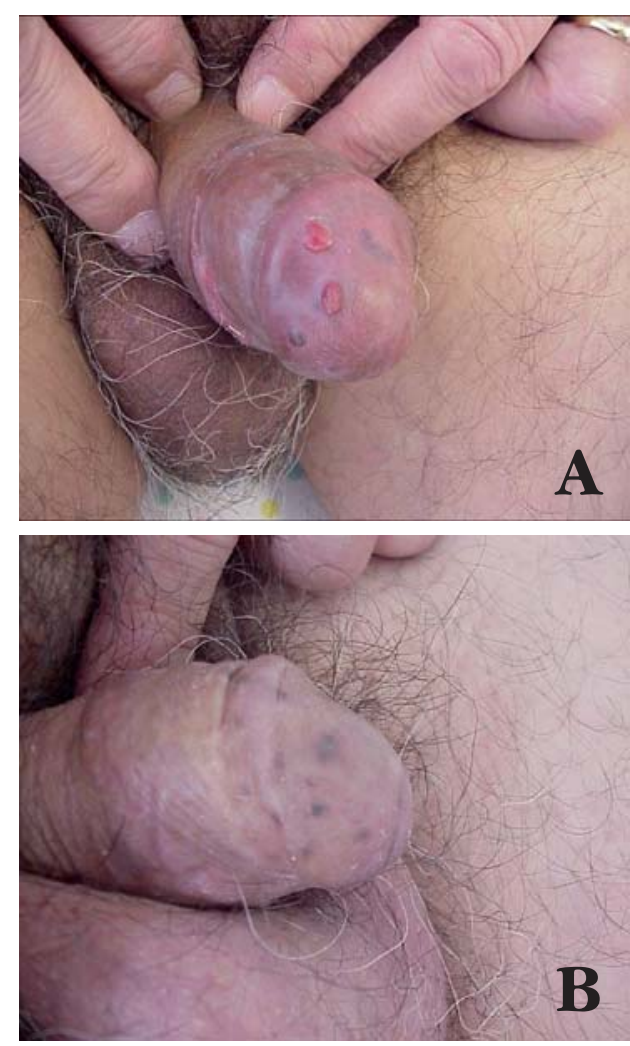

Figura 4:

A. Diminuição das lesões de pele após 20 sessões de radioterapia; B. Diminuição das lesões de pele após 40 sessões de radioterapia

A manifestação primária no pênis é rara, e mais comumente observada em pacientes HIV positivos, em que mostra um comportamento mais agressivo. ${ }^{2,3}$ Os casos desse tumor, nesta localização, em pacientes não-portadores do vírus HIV, mostram-se com pápulas, nódulos, placas e lesões verruciformes de cor eritêmato-purpúrica, geralmente assintomáticas. ${ }^{4}$ A maioria dos pacientes se apresenta com poucas lesões, embora múltiplas pápulas possam ser observa- 
das. Os locais acometidos são: a glande (mais comumente), prepúcio, sulco coronal, corpo peniano, frênulo e meato uretral. ${ }^{4,5} \mathrm{Em}$ alguns casos, relataram-se o aumento de volume do pênis e o edema linfático, pelo envolvimento maciço. ${ }^{6,7}$

O diagnóstico clínico diferencial inclui condições como: granuloma piogênico, molusco contagioso, condiloma acuminado e papulose bowenóide. ${ }^{8}$

O diagnóstico conclusivo é obtido pela biópsia. ${ }^{3} \mathrm{O}$ padrão histológico encontrado é similar ao do sarcoma de Kaposi, visto nas demais áreas do corpo.

A natureza exata da doença permanece desconhecida. Atualmente cogita-se a associação do sarcoma de Kaposi ao vírus herpes humano 8 (HHV-8), que é transmitido sexualmente, por via sanguínea ou pela saliva. ${ }^{9,10}$

Para tratamento da forma clássica, são descritos a excisão cirúrgica local, crioterapia, eletrocirurgia, quimioterapia, laserterapia e radioterapia, além de tratamento adjuvante com injeções intralesionais ou sistêmicos com citostáticos, alfa e beta- interferon. ${ }^{4} \mathrm{~A}$

\section{REFERÊNCIAS}

1. Morelli L, Pusiol T, Piscioli F, Hofler H, Weirich G, Werner M, et al. Herpesvirus 8-associated penile Kaposi's sarcoma in an HIV-negative patient: first report of a solitary lesion. Am J Dermatopathol. 2003;25:28-31.

2. Bayne D, Wise GJ. Kaposi sarcoma of penis and genitalia: a disease of our times. Urology. 1988;31:22-5.

3. Chitale SV, Peat D, Meaden JD, Johnson HB, Burgess NA. Kaposi's sarcoma of the glans penis in an HIV negative patient. Int Urol Nephrol. 2002;34:251-3.

4. Micali G, Nasca MR, De Pasquale R, Innocenzi D. Primary classic Kaposi's sarcoma of the penis: report of a case and review. J Eur Acad Dermatol Venereol. 2003; 17:320-3

5. Ekmekci TR, Kendirci M, Kizilkaya O, Koslu A. Sildenafil citrate-aided radiotherapy for the treatment of Kaposi's sarcoma of the penis. J Eur Acad Dermatol Venereol. 2005;19:603-4.

6. Gönen M, Cenker A, Kiyici H, Kalkan M. Penile Kaposi's sarcomas in a circumcised and HIV-seronegative patient. Int J Urol. 2006;13:318-20.

7. Schwartz RA, Cohen JB, Watson RA, Gascon P, Ahkami RN, Ruszczak Z, et al. Penile Kaposi's sarcoma preceded by chronic penile lymphoedema. $\mathrm{Br} \mathrm{J}$ Dermatol. 2000;142:153-6. escolha da modalidade a ser empregada deve ser individualizada a cada caso, não havendo consensos estabelecidos. Para lesões pequenas e únicas, recomendase a excisão cirúrgica. ${ }^{8}$ Geralmente, são raras as recorrências locais, após a remoção completa da lesão primária e, quando ocorrem, surgem cerca de seis meses a dois anos, após o procedimento. ${ }^{9}$ No caso de lesões maiores e múltiplas, a melhor indicação é a radioterapia, em que as recorrências são infrequentes. Nos casos mais avançados, com envolvimento visceral, ou generalizado, a quimioterapia pode ser uma boa indicação.

A radioterapia, embora bem indicada nesse tipo de tumor, deve ser empregada com monitoração periódica e contínua. Casos de fibrose do corpo do pênis, disfunção erétil e surgimento de novas lesões nos sítios não tratados podem ocorrer. ${ }^{5,9}$

Em indivíduos imunocompetentes, o sarcoma de Kaposi, apesar de raro, deve fazer parte do diagnóstico diferencial de condições, ocorridas na região genital.

8. Pacifico A, Piccolo D, Fargnoli MC, Peris K. Kaposi's sarcoma of the glans penis in an immunocompetent patient. Eur J Dermatol. 2003;13:582-3.

9. Zargari O. Exclusive penile Kaposi's sarcoma: report of an HIV-negative man successfully treated with radiotherapy. J Eur Acad Dermatol Venereol. 2006;20:318-20.

10. Monini P, de Lellis L, Fabris M, Rigolin F, Cassai E. Kaposi's sarcoma-associated herpesvirus DN sequences in prostate tissue and human semen. N Engl J Med. 1996;334:1168-72.

\author{
ENDEREÇO PARA CORRESPONDÊNCIA / MAILING ADDRESS: \\ Jesus Rodriguez Santamaria \\ Rua General Aristides Athayde Junior, 1039 - \\ Bigorrilbo \\ 80710520 Curitiba PR \\ Tel.:/Fax: $413233-7479 / 4132337479$ \\ E-mail: jsantamaria@uol.com.br
}

